\title{
Analysis of attitude errors in GRACE range-rate residuals - a comparison between SCA1B and the reprocessed attitude fused product (SCA1B + ACC1B)
}

\author{
Sujata Goswami \\ *Institut Für Erdmessung, Schneiderberg 50, 30167, Leibniz University of Hannover, Germany
}

\begin{abstract}
The precision of the attitude in the inter-satellite ranging missions like GRACE is one of the important requirement. It is required not only for the mission performance but also for the good quality of the gravity field models which are estimated from the inter-satellite ranging measurements. Here we present a comparative study of the analysis of two attitude datasets. One of them is the standard SCA1B release 2 datasets provided by JPL NASA and another is the reprocessed attitude computed at TU Graz by combining the angular accelerations and the standard SCA1B release 2 datasets. Further we also present the impact of the attitude datasets on the inter-satellite range measurements by analyzing their residuals. Our analysis reveals the significant improvement in the attitude due to the reprocessed product and reduced value of residuals computed from the reprocessed attitude.
\end{abstract}

Index Terms-GRACE, range-rate residuals, attitude errors, attitude fusion.

\section{INTRODUCTION}

The GRACE satellite mission has successfully provided the gravity field products for more than 15 years (2002-2017). The gravity field solutions are computed from the K-band range-rate observations $(\dot{\rho})$ which measures the tiny mass changes in the Earth, with the precision in $\mu \mathrm{m}[2]$. The precision of the estimated gravity field solution computed from the range-rate measurements has not met requirements defined by [3] before the launch of the GRACE mission. This requirement is termed as GRACE baseline which is still several orders of magnitude below the current achieved precision (cf. Fig. 1 .

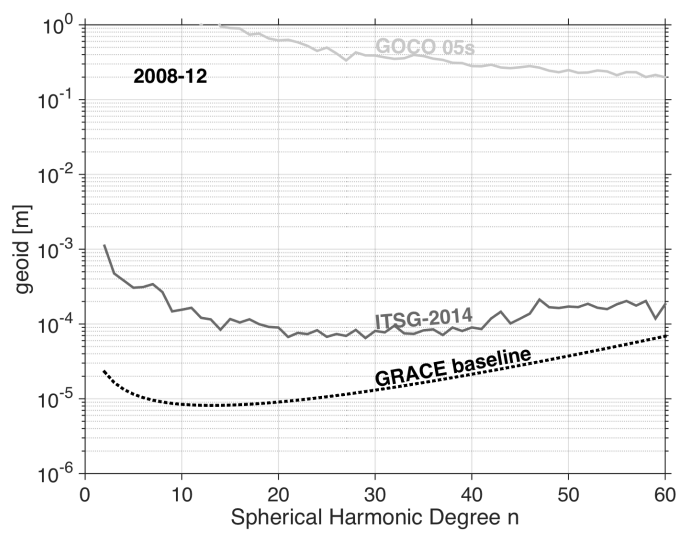

Fig. 1. Geoid degree amplitudes of the ITSG-2014 solutions compared with the GRACE baseline. The differences are presented with respect to GOCO05s static field. The gravity field solution is for the month of December 2008.

Out of many error sources responsible for this limited precision, one of the source is the errors of the attitude sensors which propagate to the estimated gravity field solutions through the $\dot{\rho}$. In order to minimize them, we must know the characteristics of those attitude errors which are affecting the precision of the $\dot{\rho}$. In the GRACE

Corresponding author: S. Goswami (e-mail: sujata.goswami@aei.mpg.de). Associate Editor: Alan Smithee.

Digital Object Identifier 10.1109/LSENS.2017.0000000 mission, so far, the model of the attitude errors is also unknown [3 which makes it even more difficult to analyze them as we do not know the frequency range where these errors dominates. One way to analyze the attitude errors in this situation would be - to compare the impact of different attitude datasets on the $\dot{\rho}$. Now, it is again difficult to analyze the direct impact of attitude errors on the $\dot{\rho}$ as they contain mass change signals and errors both, which makes it complicated to analyze the errors in details. Therefore, we analyze the attitude errors in the residuals of the $\dot{\rho}$ observations which we compute after the gravity field parameter estimation using least squares estimation as shown in Eqn. 2 [8], [14]. These range-rate residuals ( $\hat{\mathbf{e}}$ in Eqn. 2) reflect the errors which are partially absorbed by the estimated gravity field parameters $(\hat{\mathbf{x}})$. Thus, their analysis is a good basis to understand the attitude errors affecting the range-rate observations and the gravity field parameters. Therefore, in this contribution our aim is to present the results on -

$A$. An analysis of the attitude error characteristics by comparing the two different attitude datasets

$B$. The propagation of these errors into the K-band range-rate observations by analyzing their residuals.

\section{Details of the attitude data used in this work}

Before discussing the results of our findings, we discuss the datasets representing the GRACE satellite attitude used in this work -

\#1 - SCA1B release 2, the standard Level1B attitude data computed from the combination of the data of the two star cameras present on each of the two spacecrafts [7]. The data of the two star cameras is combined using the algorithm described in [9].

\#2 - Reprocessed attitude (SCA1B quaternions + ACC1B angular accelerations), the set of quaternions provided in the SCA1B are combined with the angular accelerations provided in the ACC1B product. The combination details are provided in [1] For details about the Level1B products (SCA1B and ACC1B), refer to [7]. Here we present the results of the analysis of two years of GRACE data i.e. 2007 and 2008.

The attitude data is required to compute the range-rate antenna offset corrections (AOC) which are added to the K-band range-rates 
from the instrument frame to the Line of sight (LOS) frame of reference (cf. Eqn. 11. The AOC are computed as given in [13].

$$
\dot{\rho}_{\mathrm{LOS}}=\dot{\rho}_{\mathrm{KBR}}+\dot{\rho}_{\mathrm{AOC}}
$$

The attitude information is also needed to rotate the linear accelerations in $\mathrm{ACC} 1 \mathrm{~B}$ product from the science reference frame to the inertial frame of reference [7]. Thus, the attitude errors propagate to the range-rate observations via AOC and the linear accelerations which are used to reduce the effect of the drag acting on the spacecraft and then on the K-band observations. In this contribution, we discuss the propagated errors via AOC. The range-rate residuals (î) obtained after removing all the perturbations from them are used as the observations in the gravity field parameter estimation (cf. Eqn. 2). Once we estimate the gravity field parameters, the residuals ( $\hat{\mathbf{e}})$ obtained are used in this study to analyze the attitude errors.

$$
\hat{\mathbf{l}}-\mathbf{A} \hat{\mathbf{x}}=\hat{\mathbf{e}}
$$

where, $\hat{\mathbf{I}}$ are the estimated range-rate observations, $\mathbf{A}$ is the design matrix, $\hat{\mathbf{x}}$ contains the estimated gravity field parameters and $\hat{\mathbf{e}}$ are the range-rate residuals estimated after least-squares fit using the ITSG-2014 gravity field processing chain [12].

\section{RESULTS}

\section{A. Error characteristics of the two attitude datasets}

We compare the characteristics of the two attitude datasets by analyzing the differences between their pointing angles. In Fig. 2 the comparison between the power spectral densities of the pointing angles roll, pitch and yaw of the attitude data $\# \mathbf{1}$ and $\# \mathbf{2}$ shows very small differences in roll, that too in very high frequencies whereas, the pitch and yaw angles shows the deviation after $>5.5 \mathrm{mHz}$ which becomes very large after $>9 \mathrm{mHz}$. The pitch and yaw angles computed from the attitude data $\mathbf{\# 2}$ have low high frequency noise which is due to the angular accelerations combined with the star camera datasets. Thus, the low accuracy of the star camera data in high frequencies, can be complimented by combining it with the angular accelerations. Thus, we have shown that the combination of the angular accelerations with the star camera data improves the accuracy of the attitude in high frequencies.

Further we are interested in understanding the details of the characteristics of the yaw and pitch angles of attitude which are improved when star camera data is combined with the angular accelerations. In order to analyze these characteristics, we plot the observations representing the pointing angles along the argument of latitude and the time in days for the two years (i.e. 2007 and 2008). The values start from $0^{\circ}$ (bottom) to $360^{\circ}$ (top) for one complete orbit. The ticks on the $y$-axis represents - the north (NP), south poles (SP) and, the ascending AE and descending equator (DE), respectively. The $\mathrm{x}$-axis respresents the two years of time in terms of days. Further details about the argument of latitude can be found in [11].

The differences between the pitch angles computed from the data \#1 and \#2 are plotted in Fig. 3 top panel, shows that the attitude data \#2 specially complement during the time when the star camera attitude is computed from only one available star camera head as shown in Fig. 3 bottom panel. The attitude data \#1 is computed by combining the data of two star camera heads present on each satellite [9] when the data from two star cameras is available, otherwise, the

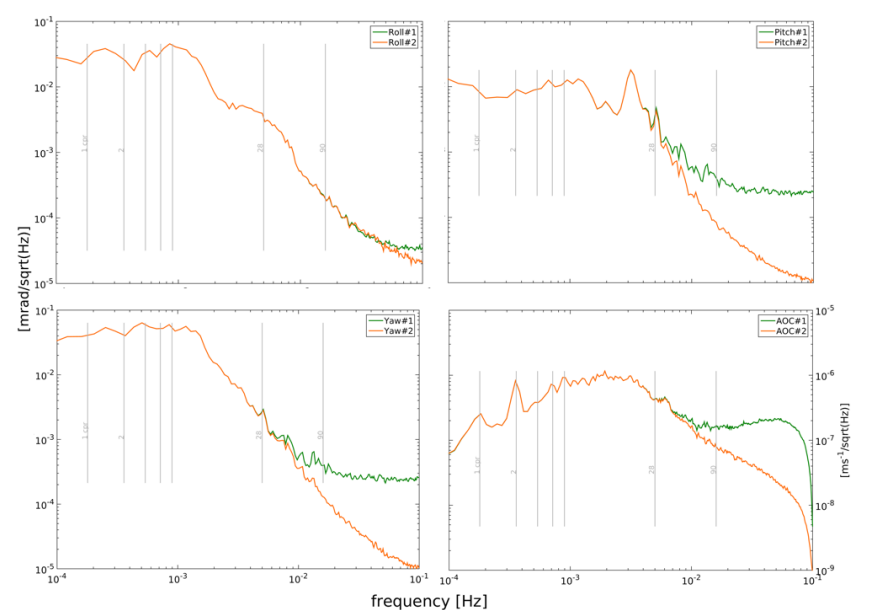

Fig. 2. PSD of the pointing angles computed from the two attitude datasets on the day 1, December 2008.

attitude used is from one of the two available star camera heads. The availability of the star camera heads on each spacecraft can be seen in Fig. 3bottom panel. The star cameras onboard gets blinded periodically by the sun (every $161 \mathrm{~d}$ ) and Moon (every $21 \mathrm{~d}$ ) intrusions into their field of view, thus, almost $50 \%$ of the time, one of the two star cameras is blinded and attitude is obtained from another available star camera. The attitude from only one star camera has high anisotropic errors as compared to the combined attitude solution [13].

The differences in the pitch angles of two GRACE satellites are exceptionally high where, the attitude \#1 is computed from only one star camera head. Thus, its combination with the angular accelerations reduces the errors significantly by providing more redundant information of the spacecraft's attitude. Therefore, the signatures related to the sun and moon intrusions into star cameras' field of view are clearly visible in the differences between the two pitch angles. Similarly, the differences between the yaw angles show the similar improved features (not shown here). These differences indicate the improvements in the high frequencies shown in the PSDs of the pointing angles shown in Fig. 2

Besides this, the time period where the attitude \#1 was affected by the attitude control actuators such as - thruster firings and changes in the currents flowing through the magnetic torquer rods (MTQs), the differences are again high in Fig. 3above. Some of the high difference places which are due to attitude actuators are marked in the Fig. 3above.

Also, we observe that the high differences shown in the pitch angles are not always consistent with the sun and moon intrusions into the star camera field of view. For example, in GRACE-A, when head\#1 was blinded from days 20 to 180 , the differences in the pitch angles were relatively high than the days from 195 to 250, where head\#2 was blinded. Based on the investigation of the accuracies of the two star camera heads onboard each spacecraft by [6], [13], we know that the attitude delivered from the head\#2 was more accurate than the head\#l on both of spacecrafts. Also, we know that there are periods where the attitude product SCA1B is computed by the data of single available star camera head. We observed that when the SCA1B was computed from the head\#2 only and this product 
is combined with the angular accelerations to produce attitude data \#2, the resulted data \#2 is more accurate than the attitude product \#2 which is computed with the combination of attitude data from head\#1 only.

Thus, we see high differences in the pitch angles when attitude product is combined with the attitude based on the SCA head\#2 and small differences when the attitude product is combined with the SCA head\#1 data. Therefore, we can say that the combination of the more accurate star camera data with the data of other sensors' (for example, angular accelerations in our case) leads to a more improved product. The accuracy of each attitude data set is also one of the important factors in the combination.

The places where the attitude SCA1B product was computed by the available attitude from the two star camera heads, its differences from the reprocessed product are small which shows that the attitude based on star camera data only is also comparably accurate provided that the data of both star camera heads is available.

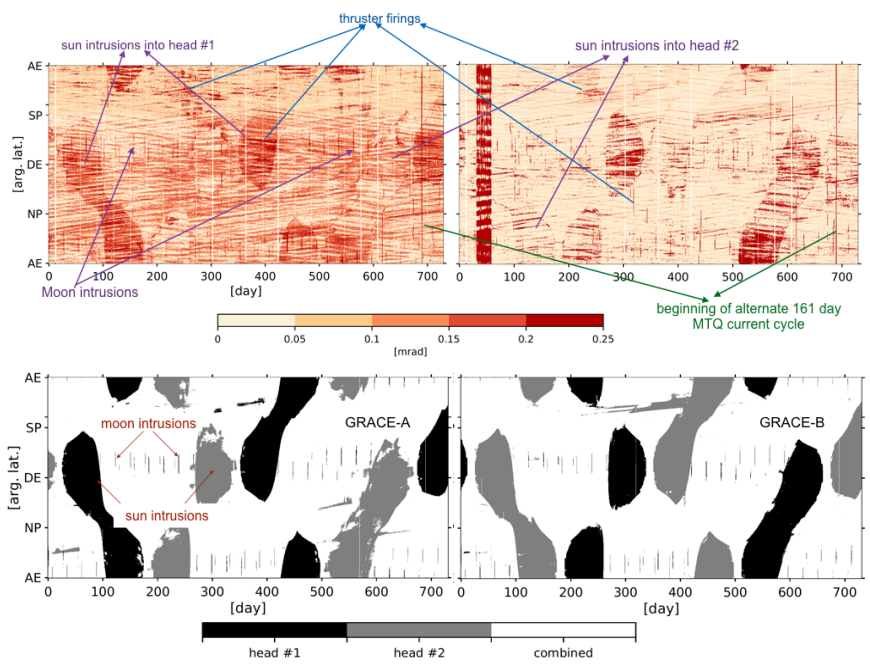

Fig. 3. top panel: Absolute differences between the pitch angles of GRACE-A and GRACE-B, computed from \#1 and \#2 respectively. bottom panel: The data flags showing the blinding status of each star camera head of GRACE-A and GRACE-B. left panel: GRACE-A and right panel: GRACE-B.

\section{B. Propagation of attitude errors to the K-band range- rate observations}

The above investigated errors in the attitude datasets propagate to the range-rate observations via AOC as shown in the Eqn. 3

$$
\mathrm{AOC}=\mathrm{PhC} \cos \phi=e_{\mathrm{AB}} \cdot\left(\mathbf{R}_{\mathrm{SRF}, \mathrm{A}}^{\mathrm{IRF}} \mathrm{PhC}_{\mathrm{A}}\right)-e_{\mathrm{AB}} \cdot\left(\mathbf{R}_{\mathrm{SRF}, \mathrm{B}}^{\mathrm{IRF}} \mathrm{PhC}_{\mathrm{B}}\right)
$$

where, $\mathrm{PhC}_{\mathrm{A}}$ and $\mathrm{PhC}_{\mathrm{B}}$ are the distance from the $\mathrm{K}$-band phase center to the satellite's center of mass, $e_{\mathrm{AB}}$ is the position vector pointing from the spacecraft A to B (cf. Fig. 4) and the rotation matrix $\mathbf{R}_{\mathrm{SRF}}^{\mathrm{IRF}}$ representing the rotation from the Science Reference Frame (SRF) to the Inertial Reference Frame (IRF), is computed from the quaternions representing attitude.

In Fig. 2, when we compare the PSDs of the pointing angles with the AOC, the differences in the two sets of AOC are visible after frequency $5.5 \mathrm{mHz}$. The two PSDs of the AOC show large deviation in the high frequencies similar to the pitch and yaw angles.

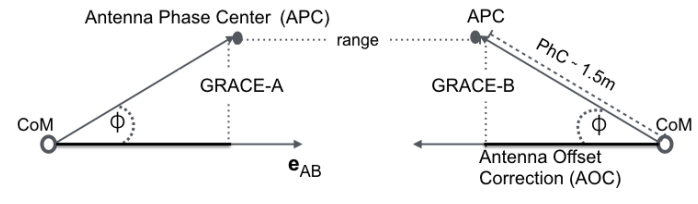

Fig. 4. A diagrammatic representation of the AOC applied to the GRACE K-band range-rate observations.

Further, to investigate the differences between two sets of AOC computed from the attitude data \#1 and \#2, we plot the observations on the argument of latitude and time plots as shown in Fig. 3 The differences between the two sets of AOC are correlated with the differences between the pointing angles of the two GRACE spacecrafts as we can see in Fig. 3top panel. In the AOC differences, we can see that the differences are high at the places when sun and moon intrudes into the star camera field of view. It indicates that the accuracy of the attitude data is highly limited by the intrusions blinding the star cameras field of view. Again, the high differences can be clearly seen and are consistent with the pitch angle differences, where the attitude is affected by the actuators actuated to control the spacecraft's attitude.

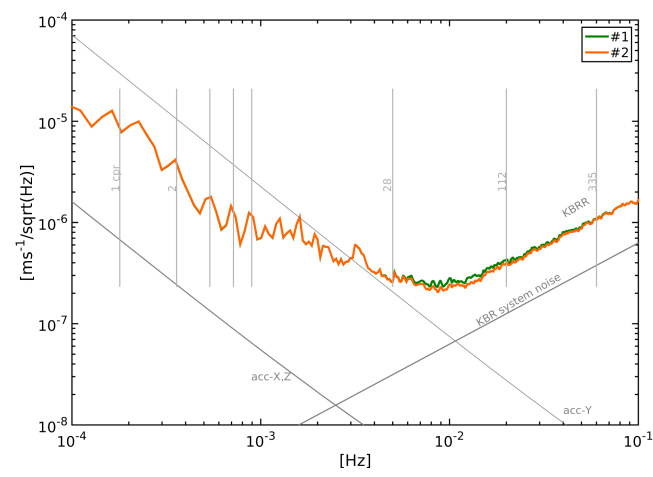

Fig. 5. A comparison of the PSD of the range-rate residuals computed from the two attitude datasets respectively.

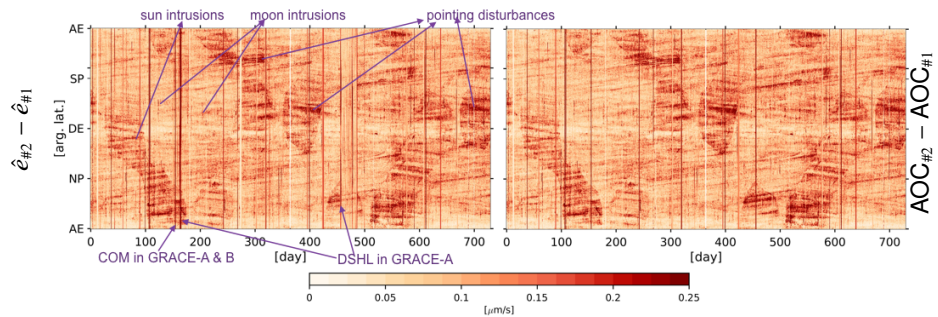

Fig. 6. left: Absolute differences between the range-rate residuals; right: Absolute differences between the AOC computed from \#1 and \#2.

There are high amplitude of residuals continuous over a full orbit, seen as vertical stripes which are mainly due to the satellite orbit and attitude control maneuvers (for example center of Mass calibration (COM), yaw axis turn, thruster firings, large magnetic torquer rod currents) and heating table related changes (so called DSHL events [5]) which indirectly affect the attitude sensors, hence, their observations. The combined attitude data $\# \mathbf{2}$ improves the attitude which is affected due to such maneuvers disturbances. 

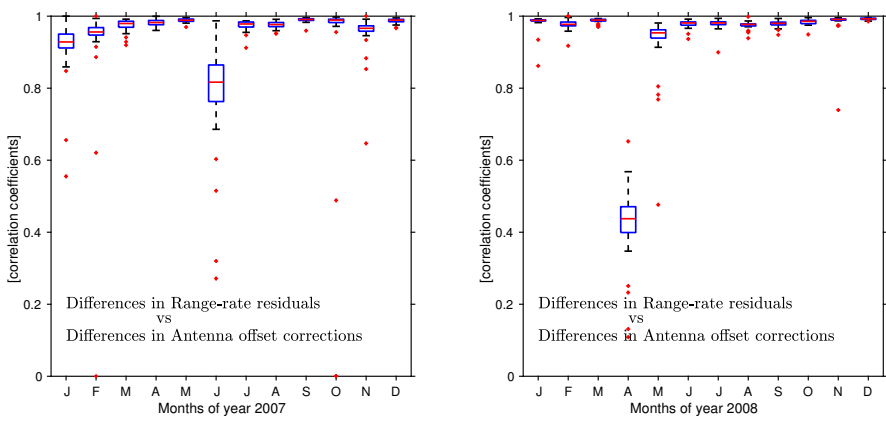

Fig. 7. Correlations between the range-rate residual differences and the AOC differences (cf. Fig. 6 in monthly box plots for the two years.

The AOC when added to the range-rate observations, propagate the attitude errors to the K-band range-rate observations (cf. Eqn. 1). The presence of these errors in the range-rate observations may affect the quality of the gravity field solutions which is the end product estimated using the range-rate observations.

An analysis of the range-rate residuals should reveal these errors, thus, indicates an insufficiency in the approach of handling the observation noise in the gravity field parameter estimation (cf. Eqn. 2). Therefore, we analyze the range-rate residuals computed after the least squares fit using each of the attitude dataset respectively. We represent the range-rate residuals as $\left(\hat{e}_{\# 1}\right)$ and $\left(\hat{e}_{\# 2}\right)$ computed from the attitude datasets \#1 and \#2 respectively. Now, when we compare the PSDs of two sets of residuals as shown in Fig. 5, we observed that the two PSDs deviate from the frequency $5.5 \mathrm{mHz}$ similar to the pitch and yaw angles. This indicates that the pitch and yaw errors are propagated to the range-rate residuals which affects the frequencies starting from $5.5 \mathrm{mHz}$. However, we do not see the large deviations in the high frequency $(>10 \mathrm{mHz})$ range-rate residuals. It is due to the noise from other known sources which is the so called KBR instrument system noise and is also the dominated noise in the residuals [4]. Thus we analyze the differences between the two set of residuals. The analysis shows the differences between the residuals are perfectly correlated with the differences of AOC between the two attitude datasets. The perfect correlations between the two sets of differences can be seen in Figs. 6 and 7respectively. The differences when plotted along the argument of latitude and time, shows that their values are high when the attitude is affected by the sun into one of the star cameras field of view and the affected attitude largely due to the actuators' actuated to control the attitude of the satellite. When we compute the correlation coefficients for daily observations for each month, we find that almost in every month the correlations between the AOC differences and residuals differences are close to 1 which shows the perfect correlation except for the month of April 2008 where correlation coefficients are very small, it may be due to the differences of residuals are propagated via the accelerometers, the discussion about it is out of scope of this paper.

\section{CONCLUSION}

We have presented the first results of the reprocessed attitude data (\#2) analysis with respect to the standard SCA1B attitude data, provided by JPL. We show that the reprocessed attitude computed by combining the angular accelerations along with the star camera datasets certainly improves the overall attitude quality. It especially compliments during the time period where the standard attiutde has been computed from one star camera data only. Also, it reduces the errors in the standard attitude where the standard attitude is affected by the attitude actuators. However, the accuracy of the star camera data is an important factor has to be considered while combining it with other attitude sensors. High accurate star cameras lead to more accurate reprocessed combined attitude which has significantly less high frequency noise as compared to the combined data computed with less accurate star camera data. Thus, we expect that the suggested improvement of the star camera data by $[10]$ and its combination with the angular accelerations will further improve the remaining errors in the GRACE attitude data.

The attitude data \#2 significantly reduces the pitch and yaw errors and correspondingly improves the AOC. We also noticed that the AOC is largely affected by the pitch and yaw pointing errors of the spaceacraft's attitude. Thus, they propagate to the range-rate observations via the AOC as shown in Eqn. 1

The pitch and yaw errors largely propagates to the residuals which are revealed by their differences. The similar magnitude of the differences between the AOC and the range-rate residuals shows that the attitude errors largely propagate via AOC which is also proved by their correlation coefficients.

\section{ACKNOWLEDGMENT}

We acknowledge support from the German Research Foundation DFG within SFB 1128 geo-Q to fund this research. We would like to thank Prof. Jakob Flury for the fruitful discussions with him in regard to this work.

\section{REFERENCES}

[1] B. Klinger and T. Mayer-Gür, Combination of GRACE star camera and angular acceleration data, EGU General Assembly, 2014.

[2] B. D. Tapley, S. Bettadpur, M. Watkins and C. Reigber, The gravity recovery and climate experiment: Mission overview and early results, Geophys.Res. Lett., Vol.31, L09607, 2004.

[3] J. Kim, Simulation Study of A Low-Low Satellite-to-Satellite Tracking Mission, Ph.D. thesis, University of Texas, Austin, USA, 2000.

[4] J. B. Thomas, An Analysis of Gravity-Field Estimation Based on Intersatellite Dual1-Way Biased Ranging, Publication 98-15, Jet Propulsion Laboratory, Pasadena, California, 1999.

[5] J. G. Beerer and F-H. Massmann, Status GRACE mission operations, JPL, In GRACE Science Team Meeting, Potsdam, Germany, 2007.

[6] J. Herman, D. Presti, A. Codazzi and C. Belle, Attitude control for GRACE: the first low-fying satellite formation, In ESA SP-548: 18th International Symposium on Space Flight Dynamics, 2004.

[7] K. Case, G. Kruizinga and S. C. Wu, GRACE Level 1B Data Product User Handbook, JPL, NASA, 2010.

[8] KR. Koch and J. Kusche, Regularization of geopotential determination from satellite data by variance components, Journal of Geodesy Vol. 76, pp. 259-268, 2002.

[9] L. Romans, Optimal combination of quaternions from multiple star cameras, JPL Internal Memorandum, 2003.

[10] N. Harvey, GRACE star camera noise, doi: 10.1016/j.asr.2016.04.025, Adv. in Sp. Res., 2016.

[11] O. Montenbruck and E. Gill, Satellite Orbits - Models, Methods and Applications, Springer-Verlag Berlin Heidelberg, XI, 369, ISBN:978-3-642- 58351-3, 2000.

[12] T. Mayer-Gürr, N. Zehentner, B. Klinger and A. Kvas ITSG-Grace2014: a new GRACE gravity field release computed in Graz, GSTM, Potsdam, Deutschland, 2014.

[13] T. Bandikova, Role of attitude determination for inter-satellite ranging, $\mathrm{Ph} . \mathrm{D}$ thesis, DGK reihe C, Nr. 758, Leibniz University of Hannover, Germany, 2015.

[14] T. Mayer-Gürr, Gravitationsfeldbestimmung aus der analyse kurzer bahnb İLogen am beispiel der satellitenmissionen CHAMP und GRACE, PhD Thesis, ITG Bonn University, Germany, 2006. 